\title{
Some moment results about the limit of a martingale related to the supercritical branching random walk and perpetuities
}

\author{
Aleksander M. Iksanov* \\ Faculty of Cybernetics, Kiev National University, \\ 01033 Kiev, Ukraine \\ Uwe Rösler ${ }^{\dagger}$ \\ Mathematisches Seminar, Christian-Albrechts-Universität zu Kiel, \\ Ludewig-Meyn-Str.4, D-24098 Kiel, Germany
}

SUMMARY. Let $\mathcal{M}^{(n)}, n=1,2, \ldots$ be the supercritical branching random walk in which the family sizes may be infinite with positive probability. Assume that a natural martingale related to the $\mathcal{M}^{(n)}$ converges almost surely and in mean to a random variable $W$. For a large subclass of nonnegative and concave functions $f$ we provide a criterion for finiteness of $\mathbb{E} W f(W)$. Our main assertions generalize some results obtained in a recent Kuhlbusch's Ph.D. thesis as well as previously known results for the GaltonWatson processes. On the way to the proof we study the existence of the $f$-moments of perpetuities.

MSC: Primary: 60J80, 60E99 ; Secondary: 60G50

Key words: branching random walk; martingale convergence; perpetuity; concave function; moments; change of measure.

*e-mail address: iksan@unicyb.kiev.ua

$\dagger^{\dagger}$ e-mail address: roesler@computerlabor.math.uni-kiel.de 


\section{Introduction and results}

Assume that an initial ancestor of some population is placed at the origin of the real line. She produces offspring who form the first generation of the population. Each individual of the first generation in her turn gives birth to children too. All children of the individuals of the first generation constitute the second generation and so on. A point process $\mathcal{M}$ with points $\infty>A_{1} \geq A_{2} \geq \ldots$ controls the location of the population over the real line in such a way. For $i=1,2, \ldots$ the displacements of the individuals of the $i$-th generation relative to positions of their mothers (they reside in the $i-1$-th generation) are given by independent copies of $\mathcal{M}$. The sequence of the point processes $\mathcal{M}^{(n)}, n=1,2, \ldots$ which define positions of the $n$-th generation individuals is called the branching random walk (the BRW, in short). Many references related to the BRW can be found in [17], [15] and [8].

In the sequel, for $n=1,2, \ldots \mathcal{F}^{n}$ denotes a $\sigma$-field containing all information about the first $n$ generations. The position of the individual $u$ is denoted by $A_{u}$; the symbol $|u|=n$ means that the individual $u$ resides in the $n$-th

generation; the symbol $\sum_{|u|=n}$ denotes the summation over all individuals of the $n$-th generation.

Set $K:=\mathcal{M}(\mathbb{R})$ and $q:=\mathbb{P}\{K<\infty\} \in[0,1]$. In this paper we only consider the supercritical BRW. Therefore, if $q=1$ we additionally assume that $\mathbb{E} K>1$. Recall that the supercriticality ensures the survival of the population with a positive probability.

Define the function

$$
m(y):=\mathbb{E} \sum_{i=1}^{K} e^{y A_{i}} \in(0, \infty], y>0 .
$$

Assume that $m(\gamma)<\infty$ for some $\gamma>0$ and set

$$
W_{n}^{(\gamma)}:=m(\gamma)^{-n} \sum_{|u|=n} e^{\gamma A_{u}}, n=1,2, \ldots
$$

The sequence $\left(W_{n}^{(\gamma)}, \mathcal{F}^{n}\right), n=1,2, \ldots$ is a nonnegative martingale (Kingman [12] and Biggins [3] were the first to study such a martingale). Since $\gamma$ and $\mathcal{F}^{n}$ will be the same from line to line, in what follows the martingale is denoted just by $W_{n}$. This martingale converges either almost surely to zero or almost 
surely and in mean to a random variable $W$ which is positive with positive probability (throughout the text we use words "positive" and "increasing" in a strict sense). Put $Y_{i}:=e^{-\gamma A_{i}} / m(\gamma)$. The (probability) distribution of $W$ satisfies the equality

$$
W \stackrel{d}{=} \sum_{i=1}^{K} Y_{i} W^{(i)}
$$

where, given $\mathcal{F}^{1}, W^{(1)}, W^{(2)}, \ldots$ are conditionally independent copies of the $W$.

The papers [3], [14], [16] provide conditions for the martingale convergence in mean. However, all these authors required more or less restrictive additional assumptions. Our Proposition 1.1 can be read from Theorem 2 [8], where the criterion of the above mentioned convergence has been obtained (but in other terms).

The equality

$$
\mathbb{E} \sum_{i=1}^{K} Y_{i} t\left(Y_{i}\right)=\mathbb{E} t(Z),
$$

which is assumed to hold for bounded Borel function $t$, defines the distribution of a random variable $Z$. Notice that

$$
\mathbb{P}\{Z=0\}=0 .
$$

In the sequel, we additionally always assume that

$$
\mathbb{P}\{Z=1\}<1, \mathbb{P}\left\{W_{1}=1\right\}<1 .
$$

As soon as the distribution of $Z$ was defined we can permit for (1) to hold for any Borel function $t$. In that case we assume that if the left-hand side is infinite or does not exist, the same is true for the right-hand side.

Let $T_{n}, n=0,1, \ldots$ be the random walk starting at zero with a step distributed like $V:=-\log Z$. Define the function

$$
A_{Z}(y):=\int_{0}^{y} \mathbb{P}\{V>x\} d x, y>0 .
$$

Relevant properties of this function can be found in [6].

Proposition 1.1. The martingale $W_{n}$ converges in mean if and only if

$$
\lim _{n \rightarrow \infty} T_{n}=+\infty \text { a.s.; } \int_{(1, \infty)} \frac{x \log x}{A_{Z}(x)} d \mathbb{P}\left\{W_{1} \leq x\right\}<\infty,
$$


or equivalently if and only if either

(i) $\mathbb{E} V \in(0, \infty)$ and $\mathbb{E} W_{1} \log ^{+} W_{1}<\infty$, or

(ii) $\mathbb{E} V=\infty$ and $\int_{(1, \infty)} \frac{x \log x}{A_{Z}(x)} d \mathbb{P}\left\{W_{1} \leq x\right\}<\infty$, or

(c) $\mathbb{E} V$ does not exist and $\mathbb{E}\left(\frac{\log ^{+} Z}{A_{Z}\left(\log ^{+} Z\right)}\right)<\infty$, and

$\int_{(1, \infty)} \frac{x \log x}{A_{Z}(x)} d \mathbb{P}\left\{W_{1} \leq x\right\}<\infty$.

Remark 1.2. In any case, the classical $x \log ^{+} x$ condition together with the condition $\lim _{n \rightarrow \infty} T_{n}=+\infty$ a.s. are sufficient for the mean convergence of the martingale. A quite remarkable fact is that when $\mathbb{E} V$ is infinite or does not exist, the $x \log ^{+} x$ condition is no longer necessary. Thus we come to a bit discouraging conclusion: the weaker moment restriction is imposed on $V$, the weaker moment condition may be put on $W_{1}$.

As soon as the problem of existence of somewhere positive $W$ is settled, it is natural to want to investigate moments of $W$. Following this principle, in this paper we will study $f$-moments of $W$. Consequently, the description of appropriate functions $f$ will be given next.

Throughout the text we assume that one of the following two assumptions is in force.

Assumption A. Function $f>0$ is nondecreasing and concave on $[0, \infty)$, $\lim _{x \rightarrow \infty} f(x)=\infty$. For fixed $B, d \geq 0$ a new function $g$ is defined by

$$
g(x):=B+\int_{d}^{x}(f(y) / y) d y, \text { for } x \geq d ; g(x)=0 \text { for } x<d .
$$

Assumption B. Function $f$ is nondecreasing and concave on $[0, \infty), \lim _{x \rightarrow \infty} f(x)=$ $\infty$ and $f(0)=0$. Additionally, there exists $p>0$ such that

$$
f(x y) \leq p f(x) f(y), \text { for all } x, y>0 .
$$

In this paper $\psi(x), x \geq 0$ is called a submultiplicative function, if $\psi(x)$ is finite, positive and Borel measurable and

$$
\psi(0)=1 \text { and } \psi(x+y) \leq \psi(x) \psi(y)
$$

Recall that for a submultiplicative function $\psi$, there exists a limit

$$
\lim _{x \rightarrow \infty} \frac{\log \psi(x)}{x} \in[0, \infty) .
$$


Inequality (3) implies that

$$
h(x+y):=f\left(e^{x+y}\right) \leq p h(x) h(y), \text { for all } x, y>0 .
$$

As pointed out by Sgibnev on page 85 of [20], the latter implies that there exists a nondecreasing submultiplicative function $\psi$ such that

$$
p_{1} \psi(x) \leq h(x) \leq p_{2} \psi(x) \text { for some positive constants } p_{1}, p_{2} .
$$

Therefore, we can define a constant $r \in[0,1]$ by

$$
r:=\lim _{x \rightarrow \infty} \frac{\log h(x)}{x} .
$$

In what follows, $F \asymp G$ means that

$$
0<\liminf _{x \rightarrow \infty} F(x) / G(x) \leq \limsup _{x \rightarrow \infty} F(x) / G(x)<\infty .
$$

We are now ready to present our first main result.

Theorem 1.3. Let $f$ satisfy the Assumption A or B.

(a) If $\mathcal{M}\left(-\infty,-\gamma^{-1} \log m(\gamma)\right)=0$ a.s., assume that the Assumption A holds. If the integral in (2) converges then

$$
\mathbb{E} W_{1} g\left(W_{1}\right)<\infty \Rightarrow \mathbb{E} W f(W)<\infty
$$

if $\mathbb{E} V \in(0, \infty)$ then

$$
\mathbb{E} W_{1} g\left(W_{1}\right)<\infty \Leftarrow \mathbb{E} W f(W)<\infty
$$

In particular, if $\mathbb{E} V \in(0, \infty)$ and $\mathbb{E} W_{1} \log ^{+} W_{1}<\infty$ then both implications hold and we have the equivalence. If $f \asymp g$ then we have the equivalence under the weaker assumption that the integral in (2) converges.

(b) If $\mathcal{M}\left(-\infty,-\gamma^{-1} \log m(\gamma)\right) \geq 1$ with positive probability, assume that the Assumption B holds. If $\mathbb{E} V \in(0,+\infty]$ then

(b1) if $r>0$ then

$$
\mathbb{E} W_{1} f\left(W_{1}\right)<\infty, \mathbb{E} Z^{r}<1 \Rightarrow \mathbb{E} W f(W)<\infty
$$

(b2) if $r=0$ then

$$
\mathbb{E} W_{1} f\left(W_{1}\right)<\infty \Rightarrow \mathbb{E} W f(W)<\infty
$$


If $\mathbb{E} V \in(0,+\infty)$ in both cases $r>0$ and $r=0$ the converse implications hold, and we in fact have the equivalence.

Remark 1.4. In case $f(x)=x^{a}, a \in(0,1]$ Theorem 1.3 reduces to the well-known equivalence

$$
\mathbb{E} W^{a+1}<\infty \Leftrightarrow \mathbb{E} W_{1}^{a+1}<\infty, \mathbb{E} Z^{a}<1
$$

(see, for example, Proposition 4 [8]).

There are many results in the spirit of Proposition 1.3 related to the Galton-Watson process (see [1] and [9] for recent developments). In the context of the branching random walk our Proposition 1.3 generalizes a statement in Section 4 [4], Corollary 10 [18], Theorems 4.4.1 and 4.5.1 [13]. The best previously known results like our Proposition 1.3 were recently obtained in Kuhlbusch's PhD thesis [13]. In Section 2 we partially compare our results to Kuhlbusch's ones.

The technique developed in this work is an extension of the approach proposed in [9] for the case of the Galton-Watson processes and in [8]. It should be noted that independently and at the same time a similar technique has also been used in [7] in the context of branching diffusions. Our method of proof consists in comparing (under the appropriate change of measure proposed in [16]) the random variable $W$ with a so called perpetuity. Keeping this in mind we find it useful to study the existence of the $f$-moments of perpetuities.

Let $\left(Q_{1}, M_{1}\right),\left(Q_{2}, M_{2}\right), \ldots$ be independent copies of a random vector $(Q, M)$. Let $Z_{0}$ be a random variable which is independent of $(Q, M)$. We will assume throughout that

$$
\mathbb{P}\{M=0\}=0, \mathbb{P}\{Q=0\}<1 .
$$

Set $\Pi_{0}:=1$ and $\Pi_{n}:=M_{1} M_{2} \cdots M_{n}, n=1,2, \ldots ; X:=-\ln |M|$, $A_{M}(y):=\int_{0}^{y} \mathbb{P}\{X>x\} d x, y>0$. The following proposition is a selection from Theorem 2.1 [6].

Proposition 1.5. The following assertions are equivalent:

$$
\begin{aligned}
\lim _{n \rightarrow \infty} \Pi_{n}=0 \text { a.s., } & \int_{1}^{\infty}\left(\frac{\ln q}{A_{M}(\ln q)}\right) d \mathbb{P}\{|Q| \leq q\}<\infty \\
& \sum_{n=1}^{\infty}\left|\Pi_{n-1} Q_{n}\right|<\infty \quad \text { a.s. }
\end{aligned}
$$


Each of these ensures

$$
\lim _{n \rightarrow \infty}\left(\sum_{k=1}^{n} \Pi_{n-1} Q_{n}+\Pi_{n} Z_{0}\right)=Z_{\infty} \text { a.s. }
$$

where

$$
Z_{\infty}:=\sum_{n=1}^{\infty} \Pi_{n-1} Q_{n} .
$$

In the literature there exist several results about the existence of moments (or the tail behaviour) of the random variable $Z_{\infty}$ called a perpetuity. We only mention two of them.

(a) $\mathbb{E}|M|^{p}<1$ and $\mathbb{E}|Q|^{p}<\infty \Leftrightarrow \mathbb{E}\left|Z_{\infty}\right|<\infty, p>0$ (in [11] this has been shown in case $M, Q \geq 0$, in [21] the implication $\Rightarrow$ has been proved in case $p>1)$

(b) if $\mathbb{P}\{|M| \leq 1\}=1$ and $\mathbb{E} e^{\epsilon|Q|}<\infty$ for some $\epsilon>0$, then $\mathbb{E} e^{\rho\left|Z_{\infty}\right|}<\infty$ for $0 \leq \rho<\sup \left\{\theta: \mathbb{E} e^{\theta|Q|}|M|<1\right\}$ (this fact follows from Theorem 2.1[5]; this work implicitly contains some other results related to moments).

Note that so far the existence of the $f$-moments of $Z_{\infty}$ has not been investigated (the only exception being the case $\left.f(x)=x^{a}, a \in(0,1]\right)$.

Our second main result sounds as follows.

Theorem 1.6. Let $f$ satisfy the Assumption A or B.

(a) If

$$
\mathbb{P}\{|M| \leq 1\}=1 \text { and } \mathbb{P}\{|M|=1\}<1,
$$

assume the Assumption A holds. If the integral in (5) converges then

$$
\mathbb{E} g(|Q|)<\infty \Rightarrow \mathbb{E} f\left(\left|Z_{\infty}\right|\right)<\infty
$$

if $\mathbb{E} X \in(0, \infty)$ then

$$
\mathbb{E} g(|Q|)<\infty \Leftarrow \mathbb{E} f\left(\left|Z_{\infty}\right|\right)<\infty .
$$

In particular, if $\mathbb{E} X \in(0, \infty)$ and $\mathbb{E} \log ^{+}|Q|<\infty$ then both implications hold and we have the equivalence. If $f \asymp g$ then we have the equivalence under the weaker assumption that the integral in (5) converges.

(b) If $\mathbb{P}\{|M|>1\}>0$, assume that the Assumption B holds. If $\mathbb{E} X \in$ $(0,+\infty]$ then

(b1) if $r>0$ then

$$
\mathbb{E} f(|Q|)<\infty, \mathbb{E} f(|M| \vee 1)<\infty, \mathbb{E}|M|^{r}<1 \Rightarrow \mathbb{E} f\left(\left|Z_{\infty}\right|\right)<\infty
$$


(b2) if $r=0$ then

$$
\mathbb{E} f(|Q|)<\infty, \mathbb{E} f(|M| \vee 1)<\infty \Rightarrow \mathbb{E} f\left(\left|Z_{\infty}\right|\right)<\infty
$$

If $\mathbb{E} X \in(0,+\infty)$ in both cases $r>0$ and $r=0$ the converse implications hold, and we in fact have the equivalence.

The rest of the paper is organized as follows. Section 2 contains some relevant properties of functions $f$ and $g$. In Section 3 after giving a preliminary result we study the $f$-moments of perpetuities and prove Theorem 1.6. In Section 4 we provide a careful description of a change of measure construction and prove Theorem 1.3.

\section{Properties of functions $f$ and $g$, and exam- ples}

To give a better feeling of the results obtained, we first point out some pairs $(f, g)$ which satisfy the Assumption A. These examples are taken from Section $3[9]$. We refer the interested reader to Table 1 [22] for more examples.

(1) For $p \in(0,1)$

$$
f(x)=x^{p}, g(x)=x^{p} / p
$$

(2) for $p \in(0,1]$

$$
f(x)=e^{-1} x 1_{\{x \in[0, e)\}}+\log ^{p} x 1_{\{x \geq e\}}, g(x)=(p+1)^{-1}\left(\log ^{p+1} x-1\right) 1_{\{x \geq e\}} ;
$$

for $p>1$

$$
\begin{gathered}
f(x)=p(p-1) e^{1-p} x 1_{\left\{x \in\left[0, e^{p-1}\right)\right\}}+\left(\log ^{p} x+(p-1)^{2}\right) 1_{\left\{x \geq e^{p-1}\right\}}, \\
g(x)=\left((p+1)^{-1}\left(\log ^{p+1} x-(p-1)^{p}\right)+(p-1)^{2}(\log x-p+1)\right) 1_{\left\{x \geq e^{p-1}\right\}},
\end{gathered}
$$

therefore for $p>0$

$$
f(x) \asymp \log ^{p} x, g(x) \asymp \log ^{p+1} x ;
$$

(3) for $\beta>0$ and $c:=(\beta / e)^{\beta}-\beta$

$$
\begin{gathered}
f(x)=\beta^{\beta} e^{-\beta-\exp (\beta)} x 1_{\left\{x \in\left[0, \exp \left(e^{\beta}\right)\right)\right\}}+\left(\log ^{\beta} \log x+c\right) 1_{\left\{x \geq \exp \left(e^{\beta}\right)\right\}}, \\
g(x)=\left(\log x \log ^{\beta} \log x-\beta e^{\beta}-\beta \int_{e^{\beta}}^{\log x} \log ^{\beta-1} u d u+c\left(\log x-e^{\beta}\right)\right) 1_{\left\{x \geq e^{\beta}\right\}},
\end{gathered}
$$


therefore

$$
f(x) \asymp \log ^{\beta} \log x, g(x) \asymp \log x \log ^{\beta} \log x .
$$

As it follows from Theorems 1.3(a) and 1.6(a) it is important to know when $f \asymp g$, if $(f, g)$ satisfy the Assumption A. A simple sufficient condition for this to hold was given in Corollary 1.2 [9]: if there exists an $\alpha \in(0,1)$ such that $x^{-\alpha} f(x)$ does not decrease for large $x$ then $f \asymp g$.

Now we would like to explain the point of using the Assumption B. To prove Theorem 1.6(b) it would be highly desirable if functions $f$ possess two properties: (4) and $f(x) \asymp \int_{0}^{x}(f(u) / u) d u$. The Assumption B appears to be the weakest possible one to ensure that these properties do hold. The next lemma collects some properties of functions satisfying the Assumption B.

Lemma 2.1. Let $f$ satisfy the Assumption B. Then

(a) $f$ and $g(x):=\int_{0}^{x}(f(u) / u) d u$ satisfy the Assumption A with $B=d=0$; moreover, $f \asymp g$;

(b) $\lim _{x \rightarrow \infty} \frac{f(x)}{\log ^{\epsilon} x}=\infty$, for every $\epsilon>0$.

Proof. The first part of (a) is obvious. Let us verify that $f \asymp g$. Since $f(x) / x$ is non-increasing, we have

$$
g(x)=\int_{0}^{x}(f(u) / u) d u \geq(f(x) / x) \int_{0}^{x} d u=f(x) .
$$

Using now (3) gives

$$
g(x)=\int_{0}^{1}(f(t x) / t) d t \leq p f(x) \int_{0}^{1}(f(t) / t) d t=\operatorname{const} f(x) .
$$

From these two inequalities we obtain the needed. From the proof of Proposition 2 [18] the following result can be derived: if $H: \mathbb{R}^{+} \rightarrow \mathbb{R}^{+}$is a convex function with concave derivative, $H(0)=0$ and there exists a positive constant $c$ such that $H(x y) \leq c H(x) H(y)$ for all $x, y \in \mathbb{R}^{+}$then

$$
\lim _{x \rightarrow \infty} \frac{H(x)}{x \log ^{\epsilon} x}=\infty, \text { for every } \epsilon>0 .
$$

Set $H(x):=\int_{0}^{x} f(u) d u$. Since

$$
2^{-1} x f(x) \leq H(x) \leq x f(x),
$$

we have

$$
H(x y) \leq x y f(x y) \stackrel{(3)}{\leq} p x y f(x) f(y) \leq 4 p H(x) H(y) .
$$


Therefore, the so defined $H$ possesses all the properties described above. This gives (7) and in view of (8) the statement follows.

As was indicated in the Introduction some results related to our Theorem 1.3 were given in [13]. Kuhlbusch studied the $\phi$-moments of $W$ when $\phi$ is a regularly varying function with index $\alpha$ subject to additional restrictions. If $\alpha \in(1,2)$, his Theorems 4.5.1 and 4.4.1 are contained in our Theorem 1.3(a) and Theorem 1.3(b) correspondingly. Indeed, it is well-known that given a regularly varying function $t$ with index $\alpha \in(1,2)$ there exists a concave function $z$ such that $t(x) \asymp x z(x)$. On the other hand, a concave function need not be regularly or slowly varying. Although it is a quite obvious fact we propose a simple example (due to Professor Oleg Zakusylo) of positive, increasing and concave function which is not regularly varying. Define

$$
q(x):=2^{-k} x+2^{k+1}-3, \quad \text { if } x \in\left[4^{k}, 4^{k+1}\right), k=0,1, \ldots
$$

The first three stated properties are easily seen. To check the absence of regular variation, set $x_{n}:=4^{n}, y_{n}:=3 \cdot 4^{n}, n=1,2, \ldots$ Then $\lim _{n \rightarrow \infty} \frac{q\left(2 x_{n}\right)}{q\left(x_{n}\right)}=$ $\frac{4}{3}$ and $\lim _{n \rightarrow \infty} \frac{q\left(2 y_{n}\right)}{q\left(y_{n}\right)}=\frac{8}{5}$. Another example could be given which however requires more computations (omitted here). Take

$$
q(x):=x^{\beta}\left(1+a_{\beta} \sin (\log x)+b_{\beta} \cos (\log x)\right), \beta \in(0,1)
$$

with appropriate parameters $a_{\beta}, b_{\beta}$.

\section{Moments of perpetuities}

Let $\left(\xi_{k}, \eta_{k}\right), k=1,2, \ldots$ be independent copies of a random vector $(\xi, \eta)$. Set $R_{n}:=\xi_{1}+\cdots+\xi_{n}, n=1,2, \ldots, R_{0}:=0$. Lemma 3.1 given next is needed for the proof of Proposition 3.3. Note that this lemma generalizes Proposition 7 [2].

Lemma 3.1. Assume $\lim _{n \rightarrow \infty} R_{n}=\infty$ and $\zeta:=\sup _{k \geq 0}\left(-R_{k}+\eta_{k+1}\right)<\infty$ a.s. Then

$$
\mathbb{P}\{\zeta>x\} \geq \mathbb{P}\{\eta>x\}+\int_{-\infty}^{x} \mathbb{P}\left\{\sup _{k \geq 0}\left(-R_{k}\right)>x-y\right\} d \mathbb{P}\{\eta \leq y\}, x \in \mathbb{R} .
$$


Proof. For every $n=1,2, \ldots$ put $M_{n}:=\sup \left\{k \geq 0:-R_{k}=\max _{0 \leq l \leq n}\left(-R_{l}\right)\right\}$.

Then

$$
\zeta \geq \max _{0 \leq k \leq n}\left(-R_{k}+\eta_{k+1}\right) \geq-R_{M_{n}}+\eta_{M_{n}+1}
$$

Therefore,

$$
\begin{gathered}
\mathbb{P}\{\zeta>x\} \geq \mathbb{P}\left\{-R_{M_{n}}+\eta_{M_{n}+1}>x\right\}=\sum_{m=0}^{n} \mathbb{P}\left\{-R_{M_{n}}+\eta_{M_{n}+1}>x, M_{n}=m\right\}= \\
=\sum_{m=0}^{n} \mathbb{P}\left\{-R_{m}+\eta_{m+1}>x, M_{n}=m\right\}= \\
=\sum_{m=0}^{n} \int_{\mathbb{R}} \mathbb{P}\left\{-R_{m}>x-y, M_{n}=m\right\} d \mathbb{P}\left\{\eta_{m+1} \leq y\right\}= \\
=\int_{\mathbb{R}} \sum_{m=0}^{n} \mathbb{P}\left\{-R_{m}>x-y, M_{n}=m\right\} d \mathbb{P}\{\eta \leq y\}= \\
\int_{\mathbb{R}} \mathbb{P}\left\{\max _{0 \leq k \leq n}\left(-R_{k}\right)>x-y\right\} d \mathbb{P}\{\eta \leq y\} .
\end{gathered}
$$

Since $R_{k}$ drifts to $\infty, \sup _{k \geq 0}\left(-R_{k}\right)<\infty$ a.s. Letting $n \rightarrow \infty$ and using Fatou's lemma allow us to conclude that

$$
\begin{aligned}
& \mathbb{P}\{\zeta>x\} \geq \int_{\mathbb{R}} \mathbb{P}\left\{\sup _{k \geq 0}\left(-R_{k}\right)>x-y\right\} d \mathbb{P}\{\eta \leq y\}= \\
= & \mathbb{P}\{\eta>x\}+\int_{-\infty}^{x} \mathbb{P}\left\{\sup _{k \geq 0}\left(-R_{k}\right)>x-y\right\} d \mathbb{P}\{\eta \leq y\} .
\end{aligned}
$$

The proof is complete.

Now we are ready to give

Proof of Theorem 1.6(a). The proof goes the similar path as that of Lemma 2.1 [9] and the necessity part of Proposition 1.1 [9]. The cited paper considers the particular case $M=b \in(0,1), Q \geq 0$ a.s.

$\Rightarrow$. Assume that

$$
\mathbb{E} g(|Q|)<\infty .
$$

In view of (6), $\lim _{n \rightarrow \infty} \Pi_{n}=0$ a.s. By Proposition 1.5, this together with the assumption that the integral in (5) converges ensures that $\left|Z_{\infty}\right|<\infty$ a.s. 
Condition (9) implies $J:=\int_{d}^{\infty} \mathbb{P}\{|Q|>v\} g^{\prime}(v) d v<\infty$. By assumption, $m:=\mathbb{E}|M| \in(0,1)$. Let us check that for arbitrary $n \in \mathbb{N}$ and fixed $c>f(d)$

$$
I_{n}:=(1-m) \mathbb{E}\left(f\left|\sum_{k=1}^{n}\right| \Pi_{k-1} Q_{k}|| \vee c\right) \leq J .
$$

We have

$$
\begin{gathered}
J=\int_{d}^{\infty} \mathbb{P}\{|Q|>v\}(f(v) / v) d v \geq(i) \\
\geq(1-m) \int_{d}^{\infty} \mathbb{P}\{|Q|>v\} \mathbb{E}\left(\sum_{k=1}^{n}\left|\Pi_{k-1}\right| f^{\prime}\left(\left|\Pi_{k-1}\right| v\right)\right) d v \geq \\
\geq(1-m) \mathbb{E} \sum_{k=1}^{n} \int_{d /\left|\Pi_{k-1}\right|}^{\infty} \mathbb{P}\{|Q|>v\}\left|\Pi_{k-1}\right| f^{\prime}\left(\left|\Pi_{k-1}\right| v\right) d v \geq(i i) \\
\geq(1-m) \int_{c}^{\infty} \sum_{k=1}^{n} \mathbb{E} \mathbb{P}\left\{\left|Q_{k}\right|>f^{-1}(x) /\left|\Pi_{k-1}\right|\right\} d x= \\
=(1-m) \sum_{k=1}^{n} \int_{c}^{\infty} \mathbb{P}\left\{f\left(\left|\Pi_{k-1}\right|\left|Q_{k}\right|\right)>x\right\} d x= \\
=(1-m) \mathbb{E} \sum_{k=1}^{n}\left(f\left(\left|\Pi_{k-1}\right|\left|Q_{k}\right|\right) \vee c\right) \geq(i i i)(1-m) \mathbb{E}\left(f\left(\sum_{k=1}^{n}\left|\Pi_{k-1}\right|\left|Q_{k}\right|\right) \vee c\right) \\
\geq(1-m) \mathbb{E}\left(f\left|\sum_{k=1}^{n} \Pi_{k-1} Q_{k}\right| \vee c\right)
\end{gathered}
$$

which proves (10). Inequality $(i)$ above has been obtained as follows: $f^{\prime}$ does not increase, the sequence $\left|\Pi_{k}(\omega)\right|, k=0,1, \ldots$ does not increase and $0<\Pi_{k}(\omega) \leq 1$ a.s. Hence,

$f(v) \geq \mathbb{E} \int_{\left|\Pi_{n}\right| v}^{v} f^{\prime}(y) d y=\mathbb{E} \sum_{k=1}^{n} \int_{\left|\Pi_{k}\right| v}^{\left|\Pi_{k-1}\right| v} f^{\prime}(y) d y \geq(1-m) v \mathbb{E} \sum_{k=1}^{n}\left|\Pi_{k-1}\right| f^{\prime}\left(\left|\Pi_{k-1}\right| v\right)$.

Equality (ii) follows by change of variable $x=f\left(v\left|\Pi_{k-1}\right|\right)$. Inequality (iii) follows from the fact that the function $x \rightarrow|x|$ is subadditive, and the functions $x \rightarrow x \vee c$ and $f(x)$ are subadditive and nondecreasing. (Take for simplicity of explanation $n=2$ and set $x:=\left|Q_{1}\right|, y:=\left|M_{1}\right|\left|Q_{2}\right|$. (iii) is 
implied by the inequalities $(f(|x|) \vee c)+(f(|y|) \vee c) \geq(f(|x|)+f(|y|) \vee c) \geq$ $(f(|x+y|) \vee c) \geq(|f(x+y)| \vee c))$.

Thus, $I_{n}$ is bounded from the above by the constant $J$ that does not depend on $n$. By the assumptions of the theorem and Proposition 1.5, the series $\sum_{k=1}^{n}\left|\Pi_{k-1}\right|\left|Q_{k}\right|$ is a.s. convergent. Since $f$ is continuous then for $n \rightarrow \infty$ the sequence $f\left(\left|\sum_{k=1}^{n} \Pi_{k-1} Q_{k}\right|\right)$ converges a.s. to $f\left(\left|\sum_{k=1}^{\infty} \Pi_{k-1} Q_{k}\right|\right)$. An appeal to Fatou's lemma gives

$$
\mathbb{E} f\left(\left|Z_{\infty}\right|\right)=\mathbb{E} f\left(\left|\sum_{k=1}^{\infty} \Pi_{k-1} Q_{k}\right|\right) \leq J<\infty .
$$

$\Leftarrow$. Assume that $\mathbb{E} f\left(\left|Z_{\infty}\right|\right)<\infty$. As in [22] pp.93-94, we can prove that $\mathbb{E} f\left(\sum_{k=1}^{\infty}\left|\Pi_{k-1}\right|\left|Q_{k}\right|\right)<\infty$. Since $\sup _{k \geq 1}\left|\Pi_{k-1}\right|\left|Q_{k}\right| \leq \sum_{k=1}^{\infty}\left|\Pi_{k-1}\right|\left|Q_{k}\right|$ and $f$ does not decrease, we have

$$
\mathbb{E} f\left(\sup _{k \geq 1}\left|\Pi_{k-1}\right|\left|Q_{k}\right|\right)<\infty .
$$

Since $\left|Q_{1}\right| \leq \sup _{k \geq 1}\left|\Pi_{k-1}\right|\left|Q_{k}\right|$, the latter inequality implies that $\mathbb{E} f(|Q|)<$ $\infty$. Thus if $f \asymp g$, combining this observation with the previous part of the theorem we deduce that $\mathbb{E} f\left(\left|Z_{\infty}\right|\right)<\infty \Leftrightarrow \mathbb{E} f(|Q|)<\infty$ under the sole condition that the integral in (5) converges.

Consider now the general case. If the support of a distribution of $Q$ is bounded, then $\left|Z_{\infty}\right|$ has finite moments of all positive integer orders, and the result of the theorem is trivial. Hence in what follows we assume that the support of a distribution of $|Q|$ is unbounded from the above. In that case there exists an $s>d$ such that $\mathbb{P}\{|Q|>t\}>0$ for all $t \geq s$.

Assume that the distribution of $M$ is nondegenerate. The strong law of large numbers implies that there almost surely exists $L>0$ such that $\left|\Pi_{k}\right| \geq e^{-2 \mu k}$ for $k \geq L$, where $\mu:=\mathbb{E} X=\mathbb{E}(-\log |M|) \in(0, \infty)$. Therefore, we can choose $k_{0}<\infty$ such that $\left|\Pi_{k}\right| \geq e^{-2 \mu\left(k \vee k_{0}\right)}$ for every $k=0,1, \ldots$ Since

$$
\begin{gathered}
\sup _{k \geq 1}\left|\Pi_{k-1}\right|\left|Q_{k}\right| \geq \sup _{k \geq k_{0}+2}\left|\Pi_{k-1}\right|\left|Q_{k}\right| \geq \sup _{k \geq k_{0}+2} e^{-2 \mu(k-1)}\left|Q_{k}\right|, \\
\sup _{k \geq k_{0}+2} e^{-2 \mu(k-1)}\left|Q_{k}\right| \stackrel{d}{=} e^{-2 \mu k_{0}} \sup _{k \geq 1} e^{-2 \mu k}\left|Q_{k}\right|
\end{gathered}
$$

and $f$ does not decrease, (11) implies

$$
\mathbb{E} f\left(\sup _{k \geq 1} e^{-2 \mu k}\left|Q_{k}\right|\right)<\infty .
$$


Since $\mu \in(0, \infty)$ and $\left|Z_{\infty}\right|<\infty$ almost surely, Proposition 1.5 allows us to conclude that $\mathbb{E} \log ^{+}|Q|<\infty$. The latter in its turn implies that

$$
C:=\prod_{k=1}^{\infty} \mathbb{P}\left\{e^{-2 \mu k}\left|Q_{k}\right| \leq s\right\}>0 .
$$

Furthermore, we have for $t \geq s$

$$
\begin{aligned}
& \mathbb{P}\left\{\sup _{k \geq 1} e^{-2 \mu k}\left|Q_{k}\right|>t\right\}=\sum_{k=1}^{\infty} \mathbb{P}\left\{|Q|>e^{2 \mu k} t\right\} \prod_{j=1}^{k-1} \mathbb{P}\left\{|Q| \leq e^{2 \mu j} t\right\} \geq \\
& \geq \sum_{k=1}^{\infty} \mathbb{P}\left\{|Q|>e^{2 \mu k} t\right\} \prod_{j=1}^{k-1} \mathbb{P}\left\{|Q| \leq e^{2 \mu j} s\right\} \geq C \sum_{k=1}^{\infty} \mathbb{P}\left\{|Q|>e^{2 \mu k} t\right\} .
\end{aligned}
$$

Assume now that the distribution of $M$ is degenerate. By assumption, $\mathbb{P}\{|M|=1\}<1$. Consequently, $\mathbb{P}\{|M|=\gamma\}=1$ for some $\gamma \in(0,1)$. An easy calculation reveals that in this case the analogue of (13) holds with $e^{-2 \mu}=\gamma$

To finish the proof it suffices to show that

$$
\int_{s}^{\infty} g^{\prime}(u) \mathbb{P}\left\{e^{-2 \mu}|Q|>u\right\} d u<\infty
$$

Recall that $g^{\prime}(u)=u^{-1} f(u)$ for $u>s$. Now (14) follows from the inequalities

$$
\begin{gathered}
\infty \stackrel{(12)}{>} \int_{s}^{\infty} f^{\prime}(t) \\
\mathbb{P}\left\{\sup _{k \geq 1} e^{-2 \mu k}\left|Q_{k}\right|>t\right\} d t \stackrel{(13)}{\geq} C \int_{s}^{\infty} f^{\prime}(t) \sum_{k=1}^{\infty} \mathbb{P}\left\{e^{-2 \mu k}\left|Q_{k}\right|>t\right\} d t \geq \\
\geq \frac{C}{e^{2 \mu}-1} \int_{s}^{\infty} f^{\prime}(t) \int_{e^{2 \mu} t}^{\infty} \frac{\mathbb{P}\{|Q|>z\}}{z} d z d t \geq \\
\geq \text { const }+\frac{C}{e^{2 \mu}-1} \int_{s}^{\infty} \frac{f(t)}{t} \mathbb{P}\left\{e^{-2 \mu}|Q|>t\right\} d t
\end{gathered}
$$

The proof of Theorem 1.3(a) is complete.

For later use it is worth recording the following corollary which can be read from the previous proof.

Corollary 3.2. Assume that the Assumption A and (6) hold, and $\mathbb{E} X \in$ $(0, \infty)$ and $\mathbb{E} \log ^{+}|Q|<\infty$. Then $\mathbb{E} f\left(\sup \left|\Pi_{k-1}\right|\left|Q_{k}\right|\right)<\infty$ implies $\mathbb{E} g(|Q|)<$ 
$\infty$.

Proposition 3.3. If $f$ is a nondecreasing function then

$$
\begin{gathered}
\mathbb{E} f\left(\left|Z_{\infty}\right|\right)<\infty \Rightarrow \mathbb{E} f\left(\sup _{k \geq 1}\left|\Pi_{k-1}\right|\left|Q_{k}\right|\right)<\infty \Rightarrow \\
\mathbb{E} f\left(\sup _{n \geq 0}\left|\Pi_{n}\right|\right)<\infty, \mathbb{E} f(|Q|)<\infty \Rightarrow \mathbb{E} f(|M| \vee 1)<\infty, \mathbb{E} f(|Q|)<\infty .
\end{gathered}
$$

Proof. Assume $\mathbb{E} f\left(\left|Z_{\infty}\right|\right)<\infty$. This implies $\mathbb{E} f\left(\sum_{k=1}^{\infty}\left|\Pi_{k-1}\right|\left|Q_{k}\right|\right)<\infty$ (see pp.93-94 [22]) and therefore

$$
\mathbb{E} f\left(\sup _{k \geq 1}\left|\Pi_{k-1}\right|\left|Q_{k}\right|\right)=\mathbb{E} h\left(\sup _{k \geq 0}\left(-S_{k}+\log \left|Q_{k+1}\right|\right)\right)<\infty,
$$

where $h(x):=f\left(e^{x}\right)$ and $S_{k}:=-\log \left|\Pi_{k}\right|, k=0,1, \ldots$ is a random walk with a step distributed like $X$. Since $Z_{\infty}<\infty$ a.s., Proposition 1.5 ensures that $\lim _{k \rightarrow \infty} S_{k}=\infty$ a.s. According to Lemma 3.1, we have

$$
\begin{gathered}
\mathbb{P}\left\{\sup _{k \geq 0}\left(-S_{k}+\log \left|Q_{k+1}\right|\right)>x\right\} \geq \mathbb{P}\{\log |Q|>x\}+ \\
+\int_{-\infty}^{x} \mathbb{P}\left\{\sup _{k \geq 0}\left(-S_{k}\right)>x-y\right\} d \mathbb{P}\{\log |Q| \leq y\}, x \in \mathbb{R} .
\end{gathered}
$$

In view of (15), we have

$$
\begin{gathered}
\infty>\int_{\mathbb{R}} h^{\prime}(x) \mathbb{P}\left\{\sup _{k \geq 0}\left(-S_{k}+\log \left|Q_{k+1}\right|\right)>x\right\} d x \stackrel{(16)}{\geq} \\
\geq \int_{\mathbb{R}} h^{\prime}(x) \int_{-\infty}^{x} \mathbb{P}\left\{\sup _{k \geq 0}\left(-S_{k}\right)>x-y\right\} d \mathbb{P}\{\log |Q| \leq y\} d x= \\
=\int_{\mathbb{R}} d \mathbb{P}\{\log |Q| \leq y\} \int_{0}^{\infty} h^{\prime}(x+y) \mathbb{P}\left\{\sup _{k \geq 0}\left(-S_{k}\right)>x\right\} d x= \\
=\mathbb{E} h\left(\log U+\sup _{k \geq 0}\left(-S_{k}\right)\right)=\mathbb{E} f\left(\sup _{k \geq 0}\left|\Pi_{k}\right|\right),
\end{gathered}
$$

where $U$ is a random variable independent of $\sup _{k>0}\left|\Pi_{k}\right|$ and distributed like $|Q|$. The condition $\mathbb{E} f\left(\operatorname{Usup}_{k \geq 0}\left|\Pi_{k}\right|\right)<\infty$ implies that $\mathbb{E} f(|Q|)<\infty$ and $\mathbb{E} f\left(\sup _{k \geq 0}\left|\Pi_{k}\right|\right)<\infty$. Since $\sup _{k \geq 0}\left|\Pi_{k}\right| \geq|M| \vee 1$, we also have $\mathbb{E} f(|M| \vee 1)<\infty$, and Proposition 3.3 has been proved. 
Proposition 3.4. Assume that the Assumption B holds and $\lim _{n \rightarrow \infty} \Pi_{n}=0$ a.s. Then

$$
\mathbb{E} f\left(\left|Z_{\infty}\right|\right)<\infty \Leftarrow \mathbb{E} f(|Q|)<\infty, \mathbb{E} f\left(\sup _{0 \leq n \leq \tau-1}\left|\Pi_{n}\right|\right)<\infty,
$$

where $\tau:=\inf \left\{n \geq 1:\left|\Pi_{n}\right|<1\right\}$.

Proof. Define the random times $N_{0}:=0$,

$$
N_{i+1}:=\inf \left\{n>N_{i}:\left|\Pi_{n}\right|<\left|\Pi_{N_{i}}\right|\right\}, i=0,1, \ldots,
$$

so that $\tau=N_{1}$. Clearly, $\mathbb{E} N_{i}<\infty, i=1,2, \ldots$ For $k=1,2, \ldots$ set

$$
\begin{gathered}
M_{k}^{\prime}:=\left|M_{N_{k-1}+1}\right| \cdots\left|M_{N_{k}}\right|, \Pi_{0}^{\prime}:=0, \Pi_{k}^{\prime}:=M_{1}^{\prime} \cdots M_{k}^{\prime} \\
Q_{k}^{\prime}:=\left|Q_{N_{k-1}+1}\right|+\left|M_{N_{k-1}+1}\right|\left|Q_{N_{k-1}+2}\right|+\cdots+\left|M_{N_{k-1}+1}\right| \cdots\left|M_{N_{k}-1}\right|\left|Q_{N_{k}}\right| .
\end{gathered}
$$

Then we have that $\left(M_{k}^{\prime}, Q_{k}^{\prime}\right)$ are independent copies of $\left(\left|\Pi_{\tau}\right|, \sum_{k=1}^{\tau}\left|\Pi_{k-1}\right|\left|Q_{k}\right|\right)$ and moreover

$$
\sum_{k=1}^{\infty}\left|\Pi_{k-1}\right|\left|Q_{k}\right|=\sum_{k=1}^{\infty} \Pi_{k-1}^{\prime} Q_{k}^{\prime} .
$$

Let us show that we can use the implication $\Rightarrow$ of Theorem 1.6(a) on the vector $\left(\left|\Pi_{\tau}\right|, \sum_{k=1}^{\tau}\left|\Pi_{k-1}\right|\left|Q_{k}\right|\right)$. Since $\left|\Pi_{\tau}\right| \in(0,1)$ a.s. and $\mathbb{P}\left\{\left|\Pi_{\tau}\right|=1\right\}=0$, it remains to verify that the integral in (5) (with $(|M|,|Q|)$ replaced by $\left.\left(\left|\Pi_{\tau}\right|, \sum_{k=1}^{\tau}\left|\Pi_{k-1}\right|\left|Q_{k}\right|\right)\right)$ converges. By Lemma 2.1, $f \asymp g$, where $g(x)=$ $\int_{0}^{x}(f(u) / u) d u$, and $f$ grows more quickly than any power of logarithm. Thus if we can show that

$$
\mathbb{E} f\left(\sum_{k=1}^{\tau}\left|\Pi_{k-1}\right|\left|Q_{k}\right|\right)<\infty
$$

then

(1) (18) implies that $\mathbb{E} \log ^{+}\left(\sum_{k=1}^{\tau}\left|\Pi_{k-1}\right|\left|Q_{k}\right|\right)<\infty$; this in turn allows us to conclude that the integral in (5) converges and therefore the needed part of Theorem 1.6(a) applies,

(2) since (18) is equivalent to $\mathbb{E} g\left(\sum_{k=1}^{\tau}\left|\Pi_{k-1}\right|\left|Q_{k}\right|\right)<\infty$, by Theorem 1.6(a) (18) implies $\mathbb{E} f\left(\sum_{k=1}^{\infty} \Pi_{k-1}^{\prime} Q_{k}^{\prime}\right)<\infty$ and hence $\mathbb{E} f\left(\sum_{k=1}^{\infty}\left|\Pi_{k-1}\right|\left|Q_{k}\right|\right)<\infty$ in view of (17).

Let $U$ be a random variable distributed like $|Q|$ and independent of $\sup _{k \geq 0}\left|\Pi_{k}\right|$. We now prove (18):

$$
\mathbb{E} f\left(\sum_{k=1}^{\tau}\left|\Pi_{k-1}\right|\left|Q_{k}\right|\right) \leq \mathbb{E} \sum_{k=1}^{\tau} f\left(\left|\Pi_{k-1}\right|\left|Q_{k}\right|\right)=
$$


(subadditivity of $f$ and $\tau<\infty$ a.s.)

$$
=\sum_{n=1}^{\infty} \mathbb{E}\left(\sum_{k=1}^{n} f\left(\left|\Pi_{k-1}\right|\left|Q_{k}\right|\right)\right) 1_{\{\tau=n\}}=\sum_{n=1}^{\infty} \mathbb{E}\left(f\left(\left|\Pi_{n-1}\right|\left|Q_{n}\right|\right)\right) 1_{\{\tau>n-1\}}=
$$

(the change of order of summation is justified by the fact that all summands are nonnegative)

$$
=\sum_{n=1}^{\infty} \int_{0}^{\infty} \mathbb{E}\left(f\left(\left|\Pi_{n-1}\right| q\right) 1_{\{\tau>n-1\}}\right) d \mathbb{P}\left\{\left|Q_{n}\right| \leq q\right\}=
$$

$\left(Q_{n}\right.$ is independent of both $\Pi_{n-1}$ and $\left.1_{\{\tau>n-1\}}\right)$

$=\int_{0}^{\infty} \sum_{n=0}^{\infty} \mathbb{E} f\left(\left|\Pi_{n}\right| q\right) 1_{\{N>n\}} d \mathbb{P}\{U \leq q\}=\mathbb{E} \tau \int_{0}^{\infty} \mathbb{E} f\left(\underset{k \geq 0}{\sup }\left|\Pi_{k}\right|\right) d \mathbb{P}\{U \leq q\}=$

(this is in fact Lemma 2 of [10]:

$$
\begin{gathered}
\mathbb{E} f\left(\operatorname{sip}_{k \geq 0}\left|\Pi_{k}\right|\right)=\mathbb{E} f\left(q \exp \left(-\inf _{k \geq 0} S_{k}\right)\right)= \\
(\mathbb{E} \tau)^{-1} \sum_{n=0}^{\infty} \mathbb{E} f\left(q \exp \left(-S_{n}\right)\right) 1_{\left\{S_{0} \leq 0, \ldots, S_{n} \leq 0\right\}}=(\mathbb{E} \tau)^{-1} \sum_{n=0}^{\infty} \mathbb{E} f\left(q\left|\Pi_{n}\right|\right) 1_{\{\tau>n\}} ;
\end{gathered}
$$

Keener assumed that $\mathbb{E} S_{1}$ exists, but this condition is not needed)

$$
=\mathbb{E} \tau \mathbb{E} f\left(\sup _{k \geq 0}\left|\Pi_{k}\right|\right) \leq p \mathbb{E} \tau \mathbb{E} f(|Q|) \mathbb{E} f\left(\sup _{k \geq 0}\left|\Pi_{k}\right|\right)<\infty,
$$

(we have used (3) and the assumptions of the proposition). The proof of Proposition 3.4 is finished.

We are now ready to give

Proof of Proposition 1.6(b). $\Rightarrow$. Let $\mathbb{E} f(|Q|)<\infty, \mathbb{E} f(|M| \vee 1)<\infty$ and $\mathbb{E}|M|^{r}<1$ if $r>0$. Using Lemma 2.1(a) we conclude that $\mathbb{E} g(|M| \vee 1)<\infty$ where $g(x)=\int_{0}^{x}(f(u) / u) d u$. Let us check that

$$
\widetilde{a}:=\int_{0}^{\infty} h(x) \mathbb{P}\left\{\log ^{+}|M|>x\right\} d x<\infty,
$$

where $h(x)=f\left(e^{x}\right)$. Notice first that

$$
g\left(e^{x}\right)=\int_{-\infty}^{x} f\left(e^{u}\right) d u=\text { const }+\int_{0}^{x} f\left(e^{u}\right) d u .
$$


Further we have $\widetilde{a}=\mathbb{E} \int_{0}^{\log ^{+}|M|} f\left(e^{x}\right) d x=-$ const $+\mathbb{E} g\left(e^{\log ^{+}|M|}\right)=-$ const + $\mathbb{E} g(|M| \vee 1)<\infty$. Inequality (4) now implies that

$$
\int_{0}^{\infty} \psi(x) \mathbb{P}\left\{\log ^{+}|M|>x\right\} d x<\infty .
$$

According to Theorem 2 of [19] the latter condition together with the conditions $\mathbb{E} \log |M| \in[-\infty, 0)$ and $\mathbb{E}|M|^{r}<1$ if $r>0$ allow us to conclude that $\mathbb{E} \psi\left(\sup _{n \geq 0}\left(-S_{n}\right)\right)<\infty$. From (4) it follows that

$$
\mathbb{E} f\left(\exp _{n \geq 0}\left(\sup _{n \geq}\left(-S_{n}\right)\right)=\mathbb{E} f\left(\sup _{n \geq 0}\left|\Pi_{n}\right|\right)<\infty .\right.
$$

It remains to apply Proposition 3.4 to conclude that $\mathbb{E} f\left(\left|Z_{\infty}\right|\right)<\infty$. $\Leftarrow$. Let now $\mathbb{E} f\left(\left|Z_{\infty}\right|\right)<\infty$. By Proposition 3.3,

$$
\infty>\mathbb{E} f\left(\sup _{n \geq 0}\left|\Pi_{n}\right|\right)=\mathbb{E} h\left(\sup _{n \geq 0}\left(-S_{n}\right)\right) \text { and } \infty>\mathbb{E} f(|Q|),
$$

where $h(x)=f\left(e^{x}\right)$. In view of (4), we have

$$
\mathbb{E} \psi\left(\sup _{n \geq 0}\left(-S_{n}\right)\right)<\infty .
$$

By the assumption of the theorem, $\mathbb{E}\left(-S_{1}\right)=\mathbb{E} \log |M| \in(-\infty, 0)$. An appeal to Theorem 1 [19] allows us to conclude that

$$
\int_{0}^{\infty} \psi(x) \mathbb{P}\left\{\log ^{+}|M|>x\right\} d x<\infty .
$$

According to $(4), \widehat{b}:=\int_{0}^{\infty} h(x) \mathbb{P}\left\{\log ^{+}|M|>x\right\} d x<\infty$. But

$$
\widehat{b}=\mathbb{E} \int_{0}^{\log ^{+}|M|} f\left(e^{x}\right) d x=- \text { const }+\mathbb{E} g\left(e^{\log ^{+}|M|}\right)=- \text { const }+\mathbb{E} g(|M| \vee 1)
$$

and $f \asymp g$. Therefore, $\mathbb{E} f(|M| \vee 1)<\infty$. If $r>0$ then (19) implies $\mathbb{E}|M|^{r}<1$ (see Remark 1 [19]). The proof of Theorem 1.6(b) is finished.

\section{Proofs related to the BRW}

Let $t_{r}$ be a rooted family tree associated with a point process $\mathcal{M}$. We say that $\left(t_{r}, X\right)$ is a labelled tree if each individual (vertex) $\theta \in t_{r} \backslash\{0\}$ is assigned its 
displacement $X(\theta)$ from its parent. The BRW defines a probability measure $\mu$ on the set of labelled trees. Lyons in [16] constructed a new probability measure $\widehat{\mu}^{*}$ on the set of infinite labelled trees with distinguished rays (a ray is an infinite line of descent starting from the root). It is under this measure $\widehat{\mu}^{*}$ we can successfully estimate the martingale limit $W$ by a perpetuity from the above, and $\sup _{k \geq 0} W_{k}$ by a largest summand of a perpetuity from below.

Under $\widehat{\mu}^{*}$ the usual family tree is replaced by a size-biased tree that has a ray with a special status. This single ray is often called trunk or spine.

For $k=1,2, \ldots$ let $v_{k}$ be the individual belonging to the trunk and sitting at the $k$-th generation, $v_{0}$ is an initial ancestor; and let $A_{v_{k}, i}, i=1,2, \ldots$ be the displacements of children of $v_{k}$ from $v_{k}$. For $k=1,2, \ldots$ let $\widehat{M}_{k}$ be the random variable which gives the displacement of $v_{k}$ from her mother; and $\widehat{Q}_{k}=\sum_{i} e^{\gamma A_{v_{k-1}, i}}$. Note that, by construction, the random vectors $\left(\widehat{M}_{k}, \widehat{Q}_{k}\right), k=1,2, \ldots$ are independent and identically distributed ${ }^{1}$.

Let $\mathcal{G}$ be the $\sigma$-field generated by the reproduction of $v_{k}, k=1,2, \ldots$, i.e. by the sequence of independent point processes $\widehat{\mathcal{M}}_{k}$ with points $A_{v_{k-1}, i}, i=$ $1,2, \ldots$ Set $\widehat{\Pi}_{0}=1$ and $\widehat{\Pi}_{k}:=\widehat{M}_{1} \ldots \widehat{M}_{k}, k=1,2, \ldots$ Now we can write the two essential inequalities which in fact were found by Lyons

$$
\begin{gathered}
\mathbb{E}_{\widehat{\mu}^{*}}\left(W_{n} \mid \mathcal{G}\right) \leq \sum_{k=1}^{n} \widehat{\Pi}_{k-1} \widehat{Q}_{k} ; \\
\sup _{k \geq 0} W_{k} \geq \sup _{k \geq 0} \widehat{\Pi}_{k} \widehat{Q}_{k+1} \quad \text { under } \widehat{\mu}^{*} .
\end{gathered}
$$

Proof of the $\Rightarrow$ part of Theorem 1.3(b). Under $\widehat{\mu}^{*}, \widehat{M}_{1} \stackrel{d}{=} Z$ and $\widehat{Q}_{1}$ has the size-biased distribution corresponding to the distribution of $W_{1}$. Thus the assumptions of the theorem can be rewritten in terms of $\widehat{M}$ and $\widehat{Q}$ as follows:

$$
\begin{gathered}
\widehat{\mu}^{*}\{\widehat{M}>1\}>0, \mathbb{E}_{\widehat{\mu}^{*}} \log \widehat{M} \in[-\infty, 0), \mathbb{E}_{\widehat{\mu}^{*}} f(\widehat{Q})<\infty \\
\text { and if } r>0 \mathbb{E}_{\widehat{\mu}^{*}} \widehat{M}^{r}<1 .
\end{gathered}
$$

Since $W_{1}=\sum_{i=1}^{K} Y_{i}$, we have $Y_{i} \leq W_{1}, i=1,2, \ldots$ Hence,

$$
\mathbb{E}_{\widehat{\mu}^{*}} f(\widehat{M} \vee 1)=\mathbb{E} f(Z \vee 1)=\mathbb{E} \sum_{i=1}^{\infty} Y_{i} f\left(Y_{i} \vee 1\right) \leq \mathbb{E} W_{1} f\left(W_{1} \vee 1\right)<\infty
$$

\footnotetext{
${ }^{1}$ Note that the proof of Theorem 2 [8] gives erroneous impression that $\widehat{M}_{k}$ and $\widehat{Q}_{k}$ are independent as well. Fortunately, this gap does not affect the proof of that result.
} 
We conclude that all the assumptions of $\Rightarrow$ part of Theorem 1.6(b) hold. Therefore, the right-hand side of (20) converges $\widehat{\mu}^{*}$ a.s. to a random variable $\widehat{Z_{\infty}}$, say and $\mathbb{E}_{\widehat{\mu}^{*}} f\left(\widehat{Z_{\infty}}\right)<\infty$. From the results of Lyons it follows that $W_{n}$ converges to $W \widehat{\mu}^{*}$ a.s. (apply Fatou's lemma to (20) to conclude $\mathbb{E}_{\widehat{\mu}^{*}}\left(\liminf _{n \rightarrow \infty} W_{n} \mid \mathcal{G}\right) \leq \widehat{Z_{\infty}}$; hence $\liminf _{n \rightarrow \infty} W_{n}<\infty$ under $\widehat{\mu}^{*}$; from the change of measure construction it follows that $1 / W_{n}$ is a positive $\widehat{\mu}^{*}$ martingale with respect to an appropriate filtration; hence the statement). By Fatou's lemma, we have

$$
\mathbb{E}_{\widehat{\mu}^{*}}(W \mid \mathcal{G}) \leq \widehat{Z_{\infty}}
$$

Since $f$ is nondecreasing and concave, then using Jensen's inequality and applying the expectation operator one more results in

$$
\begin{gathered}
\mathbb{E}_{\widehat{\mu}^{*}}(f(W) \mid \mathcal{G}) \leq f\left(\mathbb{E}_{\widehat{\mu}^{*}}(W \mid \mathcal{G})\right) \leq f\left(\widehat{Z_{\infty}}\right) ; \\
\mathbb{E}_{\widehat{\mu}^{*}} f(W) \leq \mathbb{E}_{\widehat{\mu}^{*}} f\left(\widehat{Z_{\infty}}\right) .
\end{gathered}
$$

We have already proved that the right-hand side of (22) is finite. Hence, we have

$$
\mathbb{E}_{\widehat{\mu}^{*}} f(W)<\infty .
$$

Using the Laplace-Stieltjes transforms it is easily checked that $\mathbb{E} W_{n} f\left(W_{n}\right)=$ $\mathbb{E}_{\widehat{\mu}^{*}} f\left(W_{n}\right), n=1,2, \ldots$ implies

$$
\mathbb{E} W f(W)=\mathbb{E}_{\widehat{\mu}^{*}} f(W) .
$$

This completes the proof of this part of Theorem 1.3(b).

To prove Theorem 1.3(b) in the reverse direction we need a lemma. It proposes a $\widehat{\mu}^{*}$-counterpart of the inequality obtained in Lemma 2 [4].

Lemma 4.1. For each $a>0$ small enough there exists $B>1$ such that whenever $t>1$ the following inequalities hold

$$
\widehat{\mu}^{*}\{W>t\} \leq \widehat{\mu}^{*}\left\{\sup _{n \geq 0} W_{n}>t\right\} \leq B \widehat{\mu}^{*}\{W>a t\} .
$$

In particular, for any nonnegative, nondecreasing and anti-starshaped (in particular, concave) function $h$,

$$
\mathbb{E}_{\widehat{\mu}^{*}} h(W)<\infty \Leftrightarrow \mathbb{E}_{\widehat{\mu}^{*}} h\left(\sup _{n \geq 0} W_{n}\right)<\infty .
$$


Proof of Lemma 4.1. The left-hand side inequality is obvious. Let us prove the rest. It can be checked that under $\widehat{\mu}^{*}$ the following equality of distributions holds

$$
W \stackrel{d}{=} \frac{1}{m^{n}(\gamma)} e^{\gamma v_{n}} W+\frac{1}{m^{n}(\gamma)} \sum_{|u|=n}^{\prime} e^{\gamma A_{u}} V_{u}
$$

where $\sum_{|u|=n}^{\prime}$ denotes the summation over all individuals of the $n$-th generation (of the size-biased tree) but $v_{n}$; given the information about first $n$ generations in the size-biased tree $V_{u}$ are independent copies of a random variable $V$ with distribution $\mathbb{P}\{W \in d x\}$ which is also independent of $W$.

In what follows we write $\mathbb{E}$ and $\mathbb{P}$ instead of $\mathbb{E}_{\widehat{\mu}^{*}}$ and $\widehat{\mu}^{*}$. We can choose $0<b<c$ such that $r:=\mathbb{E}(V \wedge c)=\mathbb{E}(W \wedge d) \in(0,1)$. Fix any $a \in(0, r)$. Consider the events $E_{n}:=\left\{\max _{0 \leq i \leq n-1} W_{i} \leq t, W_{n}>t\right\}, n=1,2, \ldots$. Without loss of generality we can assume that the set $\{u:|u|=n\}$ is ordered in some way such that $v_{n}$ is the first individual. Keeping this in mind, denote by $\left\{\alpha_{k}, k=1,2, \ldots\right\}$ realizations of $\left\{\frac{1}{m^{n}(\gamma) W_{n}} e^{\gamma A_{u}},|u|=n\right\}$. Note that $\sum_{k} \alpha_{k}=1$. Define the event

$$
D:=\left\{\frac{1}{m^{n}(\gamma) W_{n}} e^{\gamma v_{n}}(W \wedge d)+\frac{1}{m^{n}(\gamma) W_{n}} \sum_{|u|=n}^{\prime} e^{\gamma A_{u}}\left(V_{u} \wedge c\right)>a\right\}
$$

Then almost surely

$$
\mathbb{P}\left\{D \mid \mathcal{F}^{n}\right\}=\mathbb{P}\left\{\eta:=\alpha_{1}(W \wedge d)+\sum_{k=2}^{\infty} \alpha_{k}\left(V_{k} \wedge c\right)-a>0\right\} \geq 1 / B
$$

where $1 / B:=\frac{(r-a)^{2}}{\left(\mathbb{E}(V \wedge c-a)^{2}\right) \vee\left(\mathbb{E}(W \wedge d-a)^{2}\right)} \in(0,1)$. To get the latter inequality we have used

$$
\mathbb{P}\{\eta>0\} \geq \frac{(\mathbb{E} \eta)^{2}}{\mathbb{E} \eta^{2}}
$$

which is applicable as $\mathbb{E} \eta=r-a>0$. $\mathbb{E} \eta^{2}$ is estimated as follows:

$\mathbb{E} \eta^{2}=\alpha_{1}^{2} \mathbb{E}(W \wedge d-a)^{2}+\mathbb{E}(V \wedge c-a)^{2} \sum_{k=2}^{\infty} \alpha_{k}^{2}+2(\mathbb{E}(V \wedge c-a))^{2} \sum_{1 \leq i<j} \alpha_{i} \alpha_{j} \leq$ 


$$
\begin{gathered}
\leq\left(\left(\mathbb{E}(W \wedge d-a)^{2}\right) \vee\left(\mathbb{E}(V \wedge c-a)^{2}\right)\right) \sum_{k=1}^{\infty} \alpha_{k}^{2}+2(\mathbb{E}(V \wedge c-a))^{2} \sum_{1 \leq i<j} \alpha_{i} \alpha_{j} \leq \\
\leq\left(\left(\mathbb{E}(W \wedge d-a)^{2}\right) \vee\left(\mathbb{E}(V \wedge c-a)^{2}\right)\right)\left(\sum_{k=1}^{\infty} \alpha_{k}^{2}+2 \sum_{1 \leq i<j} \alpha_{i} \alpha_{j}\right)= \\
=\left(\left(\mathbb{E}(W \wedge d-a)^{2}\right) \vee\left(\mathbb{E}(V \wedge c-a)^{2}\right)\right) .
\end{gathered}
$$

Since $E_{n} \in \mathcal{F}^{n}$, we have $\mathbb{P}\left\{D \bigcap E_{n}\right\}=\mathbb{E} \mathbb{P}\left\{D \mid \mathcal{F}^{n}\right\} 1_{E_{n}} \geq(1 / B) \mathbb{P}\left\{E_{n}\right\}$. If $\mathbb{P}\left\{E_{n}\right\} \neq 0$, the latter implies

$$
\mathbb{P}\left\{D \mid E_{n}\right\} \geq 1 / B
$$

For $t>1$ we have

$$
\begin{gathered}
\mathbb{P}\left\{W>a t \mid E_{n}\right\} \stackrel{(24)}{\geq} \mathbb{P}\left\{\frac{1}{m^{n}(\gamma) W_{n}} e^{\gamma v_{n}} W+\frac{1}{m^{n}(\gamma) W_{n}} \sum_{|u|=n}^{\prime} e^{\gamma A_{u}} V_{u}>\frac{a t}{W_{n}} \mid E_{n}\right\} \geq \\
\geq \mathbb{P}\left\{D \mid E_{n}\right\} \stackrel{(25)}{\geq} 1 / B .
\end{gathered}
$$

The inequality

$$
\mathbb{P}\{W>a t\} \geq \sum_{n=1}^{\infty} \mathbb{P}\left\{W>a t \mid E_{n}\right\} \mathbb{P}\left\{E_{n}\right\} \geq(1 / B) \mathbb{P}\left\{\sup _{n \geq 0} W_{n}>t\right\}
$$

completes the proof of the first part of Lemma 4.1. To prove the second part we should only note that the implication $\Rightarrow$ follows from the inequality $\mathbb{E} h\left(\sup _{n \geq 0} W_{n}\right) \leq B \mathbb{E} h(W / a) \leq(B / a) \mathbb{E} h(W)$.

Proof of the $\Leftarrow$ part of Theorem 1.3(b). Assume now that $\mathbb{E} \log Z \in$ $(-\infty, 0)$ and $\mathbb{E} W f(W)<\infty$. Then $\mathbb{E}_{\widehat{\mu}^{*}} \log \widehat{M} \in(-\infty, 0)$ and in view of $(23)$, $\mathbb{E}_{\widehat{\mu}^{*}} f(W)<\infty$. Therefore, by Lemma $4.1, \mathbb{E}_{\widehat{\mu}^{*}} f\left(\sup _{k \geq 0} W_{k}\right)<\infty$. In view of (21),

$$
\infty>\mathbb{E}_{\widehat{\mu}^{*}} f\left(\sup _{k \geq 0} W_{k}\right) \geq \mathbb{E}_{\widehat{\mu}^{*}} f\left(\sup _{k \geq 0} \widehat{\Pi}_{k} \widehat{Q}_{k+1}\right) .
$$

Let us now apply Proposition 3.3 on the pair $(\widehat{M}, \widehat{Q})$ to get $\mathbb{E}_{\widehat{\mu}^{*}} f\left(\sup _{n \geq 0} \widehat{\Pi}_{n}\right)<\infty$ and $\mathbb{E}_{\widehat{\mu}^{*}} f(\widehat{Q})<\infty$. Exactly the same analysis as in the proof of Theorem 
1.3(b) (implication $\Leftarrow)$ shows that $\mathbb{E}_{\widehat{\mu}^{*}} f(\widehat{M} \vee 1)<\infty$ and if $r>0$ then $\mathbb{E}_{\widehat{\mu}^{*}} \widehat{M}^{r}<1$. It remains to recall that

$$
\mathbb{E}_{\widehat{\mu}^{*}} f(\widehat{Q})=\mathbb{E} W_{1} f\left(W_{1}\right), \mathbb{E}_{\widehat{\mu}^{*}} f(\widehat{M} \vee 1)=\mathbb{E} f(Z \vee 1), \mathbb{E}_{\widehat{\mu}^{*}} \widehat{M}^{r}=\mathbb{E} Z^{r}
$$

However, the condition $\mathbb{E} f(Z \vee 1)<\infty$ can be omitted as it is implied by $\mathbb{E} W_{1} f\left(W_{1}\right)<\infty$. The proof is complete.

Proof of Theorem 1.3(a) goes the similar but simpler way as that of Theorem 1.3(b). The only difference is that while Theorem 1.3(b) uses Theorem 1.6(b) and Proposition 3.3, Theorem 1.3(a) appeals to Theorem 1.6(a) and Corollary 3.2. We can use these statements as the condition $\mathcal{M}\left(-\infty,-\gamma^{-1} \log m(\gamma)\right)=0$ a.s. implies that the distribution of the random variable $Z$ (and hence of $\widehat{M}$ ) is concentrated on $[0,1]$. Recall that throughout the paper we assumed that $\mathbb{P}\{Z=1\}<1$.

Acknowledgement. A part of this work was done while A.M.Iksanov was visiting Kiel in December 2004. He gratefully acknowledges the hospitality and financial support received from Christian-Albrechts-Universität zu Kiel.

\section{References}

[1] Alsmeyer, G., Rösler, U., 2004. On the existence of $\phi$-moments of the limit of a normalized supercritical Galton-Watson process, J. Theor. Prob. 17, 905-928.

[2] Araman, V.F., Glynn, P.W., 2004. Tail asymptotics for the maximum of perturbed random walk. Preprint. Available online at http://pages.stern.nyu.edu/ ${ }_{\text {varaman }}$

[3] Biggins, J.D., 1977. Martingale convergence in the branching random walk, J. Appl. Prob. 14, 25-37.

[4] Biggins, J.D., 1979. Growth rates in the branching random walk, Z.Wahrsch. Gebiete. 48, 17-34.

[5] Goldie, C.M., Grübel, R. 1996. Perpetuities with thin tails, Adv.Appl.Prob. 28, 463-480.

[6] Goldie, C.M., Maller, R.A., 2000. Stability of perpetuities. Ann. Prob. 28, 1195-1218. 
[7] Hardy, R., Harris, S.C. 2004. Spine proofs for $L_{p^{-}}$ convergence of branching-diffusion martingales. Mathematics Preprint. 0405, University of Bath. Available online at http://www.bath.ac.uk/ massch/Research/Papers/spine-Lp-cgce.pdf

[8] Iksanov, A.M., 2004. Elementary fixed points of the BRW smoothing transforms with infinite number of summands. Stoch.Proc.Appl. 114, $27-50$.

[9] Iksanov, A.M., 2004. On some moments of the limit random variable for a normalized supercritical Galton-Watson process, Focus on Probability Theory, Nova Science Publishers, Inc., Commack, NY, in press.

[10] Keener, R., 1987. A note on the variance of a stopping time, Ann.Stat. 15, 1709-1712.

[11] Kellerer, H.G., 1992. Ergodic behaviour of affine recursions III:positive recurrence and null recurrence. Technical reports, Math. Inst. Univ. München, Theresienstrasse 39, D-8000 München, Germany.

[12] Kingman, J.F.C., 1975. The first birth problem for an age-dependent branching process. Ann. Prob. 3, 790-801.

[13] Kuhlbusch, D., 2004. Moment conditions for weighted branching processes. PhD thesis, Universität Münster.

[14] Liu, Q., 1997. Sur une équation fonctionnelle et ses applications: une extension du théorème de Kesten-Stigum concernant des processus de branchement. Adv.Appl.Prob. 29, 353-373.

[15] Liu, Q., 1998. Fixed points of a generalized smoothing transformation and applications to the branching random walk. Adv.Appl.Prob. 30, 85112.

[16] Lyons, R., 1997. A simple path to Biggins's martingale convergence for branching random walk. In Athreya, K.B., Jagers, P. (eds.). Classical and Modern Branching Processes, IMA Volumes in Mathematics and its Applications, vol. 84, Springer, Berlin, pp. 217-221.

[17] Rösler, U., 1992. A fixed point theorem for distributions. Stochastic Process. Appl. 42, 195-214. 
[18] Rösler, U., Topchii, V.A., Vatutin, V.A., 2000. Convergence conditions for the weighted branching process. Discrete Mathematics and Applications, 10, 5-21.

[19] Sgibnev, M.S., 1997. Submultiplicative moments of the supremum of a random walk with negative drift. Stat.Prob. Letters. 32, 377-383.

[20] Sgibnev, M.S., 1999. On the existence of submultiplicative moments for the stationary distributions of some markovian random walks. J.Appl. Prob. 36, 78-85.

[21] Vervaat, W., 1979. On a stochastic difference equation and a representation of non-negative infinitely divisible random variables. Adv. Appl. Prob. 11, 750-783.

[22] Zerner, M.P.F., 2002. Integrability of infinite sums of heavy-tailed i.i.d. random variables. Stoch.Proc.Appl. 99, 81-94. 